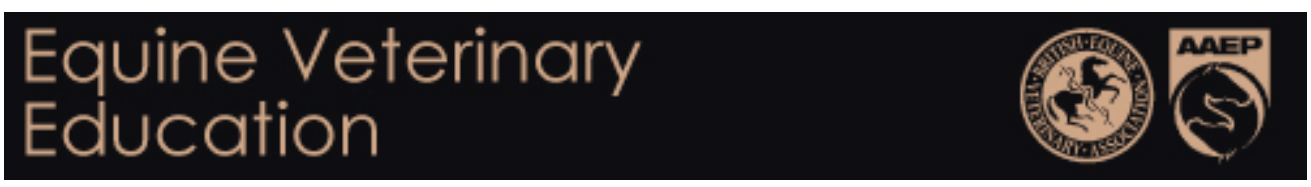

\title{
The known unknowns of equine mammary neoplasia
}

\begin{tabular}{|c|c|}
\hline Journal: & Equine Veterinary Education \\
\hline Manuscript ID & Draft \\
\hline Wiley - Manuscript type: & Clinical Commentary (requested) \\
\hline $\begin{array}{r}\text { Date Submitted by the } \\
\text { Author: }\end{array}$ & $n / a$ \\
\hline Complete List of Authors: & $\begin{array}{l}\text { Hughes, Katherine; University of Cambridge School of the Biological } \\
\text { Sciences, Veterinary Medicine }\end{array}$ \\
\hline Discipline: & Pathology \\
\hline Body System/Disorder: & Reproductive \\
\hline Abstract: & Not applicable: clinical commentary \\
\hline
\end{tabular}


3

4

5

6

7

8

9

10

11

12

13

14

15

16

17

18

19

20

The known unknowns of equine mammary neoplasia

Running title: The known unknowns of equine mammary neoplasia

Department of Veterinary Medicine, University of Cambridge, Madingley Road, Cambridge, Cambridgeshire. CB3 0ES.

* Corresponding author: kh387@,cam.ac.uk

Katherine Hughes*

20 Keywords: carcinoma; horse; mammary gland; mastitis; tumour 
Introduction

22

23

24

Primary equine mammary tumours are rare entities that are considered to have a poor prognosis (Shank, 2009). In the case report associated with this clinical commentary, the authors describe the clinical presentation, management, and histopathological assessment of a comedocarcinoma arising in the mammary gland of a 20 year-old Arabian mare (Sabiza et al., In press). In their article, the authors allude to some of the challenges of diagnosis and prognostication in cases of equine mammary neoplasia. In spite of a number of detailed descriptions of individual cases or case series of equine mammary tumours, there are numerous unanswered questions regarding carcinomas arising in the equine mammary gland, and the concept of 'known unknowns' springs to mind. This clinical commentary will consider a selection of interesting and challenging 'known unknowns' relating to the pathogenesis, diagnosis, and prognosis of equine mammary tumours.

The relationship between mammary tumourigenesis and mare reproductive history is

\section{currently undetermined}

At the time of presentation for investigation of mammary enlargement and bleeding from the teat, the mare in the associated case report was lactating (Sabiza et al., In press). This highlights an interesting question regarding the role, if any, of parity as a risk factor for equine mammary tumour development. In dogs and cats, the currently available data suggest that parity is not a risk factor for mammary tumourigenesis (Taylor et al., 1976; Overley et al., 2005). By contrast, in humans, there is an elevated breast cancer risk associated with lifetime nulliparity, and for women who have had their first childbirth at a more advanced age. Thus, for younger women having a full-term pregnancy has a relative 'protective' effect against breast cancer, quantified as a 50\% reduced risk for women who had their first fullterm pregnancy whilst under 20 years old (Subramani and Lakshmanaswamy, 2017). 
46 Childbirth at any age is associated with a transiently increased breast cancer risk and, in

47 essence, this phase of elevated risk extends for women who are older at the time of their first full-term pregnancy (Borges et al., 2020).

49

50 Identification of such patterns requires large and sophisticated epidemiological studies 51 (Lambe et al., 1994), and execution of similar veterinary investigations is often challenging. The associated difficulties are compounded and elevated in the case of rare veterinary tumour types where limited numbers of cases are recorded. Furthermore, in many cases, equine

54 patients will have multiple owners over the course of a lifetime, and a complete reproductive 55 history may be unavailable to the attending veterinarian, and cannot be accurately 56 documented for research purposes. At present, there is therefore insufficient evidence available to interrogate any potential relationship between mammary tumourigenesis and parity in the mare.

Initial clinical distinction between mastitis and mammary neoplasia may be challenging

In the mare, clinical distinction between mastitis and mammary neoplasia may be difficult (Reppas et al., 1996; Kato et al., 1998; Hirayama et al., 2003; Shank, 2009; Hughes et al., 2015; Sabiza et al., In press). Indeed, it has been suggested that horse owners and those caring for horses may associate any form of mammary gland enlargement with mastitis (Canisso et al., 2019). The presence of discharge from mammary tumours, potentially associated with draining sinus tracts, necrosis, and/or secondary infection, may complicate the clinical picture. Whilst non-specific clinical signs such as ventral or hind limb oedema are unhelpful and may be associated with a diagnosis of a mammary tumour (Munson, 1987) or mastitis (McCue and Wilson, 1989), some authors have postulated that ulceration of the overlying skin may be more indicative of a mammary tumour than of mastitis (Prendergast et 
71 al., 1999). The two conditions may also occur in tandem, with mastitis arising as a 72 consequence of the presence of mammary neoplasia.

73

74 An erroneous diagnosis of primary mastitis may lengthen the time to correct diagnosis of a 75 mammary tumour and may consequently delay surgical removal of the mass, if this treatment 76 is selected as appropriate (Shank, 2009). In this regard, cytology can be a valuable tool in 77 distinguishing some cases of mastitis from cases of mammary neoplasia. Although the secretions from a mastitic mamma have an inconsistent composition and may therefore appear cytologically normal in some cases, in other instances mastitic secretions are characterised by the presence of viable and degenerate neutrophils, necrosis and/or other degenerate debris. By contrast, cytology from neoplastic masses may reveal an abnormal population of cells exhibiting criteria of malignancy although, again, in some cases it is not helpful (Freeman, 2002; Shank, 2009). The distinction is not absolute, and, in general, chronic inflammatory processes may also result in a degree of cytological cellular atypia. Nonetheless, cytology may be a valuable tool in assessment of mammary enlargement and discharge.

87

A specific framework for phenotyping equine mammary tumours is yet to be established

90 Surgical excision of mammary masses in mares has been suggested to be the most viable

91 therapeutic modality in appropriately selected surgical candidates (Sysel et al., 1993; Shank, 92 2009). In the associated clinical report, a radical mastectomy was undertaken and subsequently histological sections from the mass were examined (Sabiza et al., In press).

94 Currently, there is no classification system for the morphological diagnosis of equine 
mammary tumours, although veterinary pathologists tend to employ morphological diagnoses similar to those used in canine and feline systems (Goldschmidt et al., 2017).

Most primary mammary tumours are carcinomas and adenocarcinomas of epithelial origin, and therefore express pan-cytokeratin, as in the case described in the associated report (Sabiza et al., In press). In the non-neoplastic mammary gland, the epithelial component comprises a bilayer (Fig 1 and 2) and the luminal and basal epithelia are distinguished by differing expression patterns of specific cytokeratins. Consequently, expression patterns of specific cytokeratins can potentially be employed to identify carcinomas of luminal and basal origin.

Cytokeratins 8 and 18 are two exclusively luminal markers in the human breast (Dontu and Ince, 2015) and cytokeratin 8 has previously been demonstrated to be similarly expressed in non-neoplastic equine mammary luminal epithelia (Hirayama et al., 2003). Cytokeratin 18 expression has been reported to be negative in frozen sections of normal equine mammary gland (Bussche et al., 2017). We have detected weak cytokeratin 8/18 expression in luminal epithelial cells using sections from formalin-fixed, paraffin-embedded tissue (Fig. 1). An equine mammary carcinoma expressing cytokeratin 18 has been described (Bussche et al., 2017).

Cytokeratin 14 is frequently used as a marker of basal, or myoepithelial, cells in the adult mouse (Gusterson and Eaves, 2018). In humans cytokeratin 14 is not exclusively expressed by basal epithelia, being expressed in basal epithelia in large ducts but in the luminal layer in lobules (Dontu and Ince, 2015). In the horse, cytokeratin 14 appears to be expressed predominantly in the basal compartment (Fig. 2) (Hirayama et al., 2003; Hughes et al., 2015) 
and mammary carcinomas expressing cytokeratin 14 have been described (Hughes et al., 2015). Alpha-smooth muscle actin is also expressed by most mammary basal epithelial cells, and it confers the contractile properties required for the expulsion of milk (Gusterson et al., 2005; Stewart et al., 2019; Hitchcock et al., 2020). Alpha-smooth muscle actin expression has previously been demonstrated in normal equine mammary gland (Kato et al., 1998; Hirayama et al., 2003) (Fig. 1).

Taken together, these data suggest that assessment of expression of specific cytokeratins and contractile proteins such as alpha-smooth muscle actin would be a fruitful focus for further investigations delineating the histological phenotype, and cell type of origin, of equine mammary tumours. Given that commercial antibodies are most usually tested against human or murine tissues, it is vital that any such studies follow rigorous protocols and that antibodies are tested and standardised on equine tissues representing appropriate positive and negative controls (Ramos-Vara, 2017).

\section{Prognostic factors for mammary tumours are currently lacking}

In their report, Sabiza et al. evaluated tubule formation, nuclear pleomorphism, and mitotic count in histological sections from the mammary mass (Sabiza et al., In press). These morphological features reflect degree of differentiation and form the basis of the Elston and Ellis system for grading breast cancer (Elston and Ellis, 1991). However, although the Elston and Ellis system has been applied to veterinary mammary cancers, such as feline mammary neoplasia, authors studying mammary tumours in that species have recently proposed that adaptions to the grading system may provide more accurate prognostic value (Mills et al., 2015; Dagher et al., 2019). This illustrates the difficulties associated with applying a grading scheme designed for one species to the same tumour type occurring in a different species. 
145 Whilst the broad framework may be helpful, parameters, and cut-off points for numerical

146 values such as mitotic count, are likely to require modification in order to offer optimum

147 prognostic value. As noted, currently, no such grading framework exists for mammary

148 tumours arising in mares (Sabiza et al., In press).

150 In addition to morphological assessment, expression of molecular markers may be evaluated in tissue sections to guide prognostication. Currently, robust data regarding such prognostic markers is lacking for equine mammary tumours. We have previously demonstrated that a subset of equine mammary carcinomas exhibit nuclear expression of STAT3 (Hughes et al., 2015), suggesting that this transcription factor, frequently constitutively active in invasive breast cancer (Watson and Miller, 1995), might also be activated in some equine mammary tumours. However, we have yet to evaluate the use of STAT3 immunohistochemical staining as a prognostic marker for equine mammary tumours.

159 Other authors have utilised quantitative reverse transcription polymerase chain reaction to 160 demonstrate reduction in p53 expression in a single equine mammary tumour compared to adjacent non-neoplastic tissue. This observation indicates that evaluation of p53 expression in a larger set of tumours may be warranted (Bussche et al., 2017).

\section{Conclusions}

165 It has been previously noted that challenges surrounding the diagnosis and management of equine mammary tumours, particularly in remote areas, may constitute a significant psychological burden to the veterinary clinician as well as having deleterious effects on the patient's owner and/or groom (Boyce and Goodwin, 2017). Some of the known unknowns discussed here, particularly those associated with diagnosis and prognostication, contribute to 
170

171 further scientific knowledge and to provide the best outcome for our patients, constitute

172 strong drivers for continued research in this field.

173

174 Materials and methods for unpublished experiments

175 Dual immunohistochemical staining for alpha smooth muscle actin (rabbit monoclonal 176 antibody catalogue number ab124964; 1:2000) ${ }^{1}$ and keratin 8/18 (mouse monoclonal

177 antibody catalogue number \#4546; 1:50) ${ }^{2}$ was conducted using a standard protocol and an 178 ImmPRESS Duet Double Staining Polymer Kit ${ }^{3}$. Negative control slides were prepared using 179 species-matched immunoglobulins.

180

181

182

183

184

185

186

187

188

189

190

191

192

193

194

\section{Declarations}

Authorship: KH conceived and designed the clinical commentary, performed experiments, and wrote the manuscript.

Authors' declarations of interests: There are no conflicts of interest.

Funding: The author gratefully acknowledges The Pathological Society of Great Britain and Ireland for funding her current research (ICA 1019 02).

Ethical Animal Research: Owner informed consent: Sections used for immunohistochemistry were obtained from tissues that were surplus to diagnostic requirements collected from cases examined post mortem by the anatomic pathology service of the Department of Veterinary Medicine, University of Cambridge. Consent for retention of tissues for teaching and research purposes was granted at the time of submission for post mortem examination.

\section{Acknowledgements}


195 The author gratefully acknowledges the excellent technical expertise of Debbie Sabin in the 196 preparation of unstained tissue sections for immunohistochemistry. The author apologises to 197 all investigators whose work unfortunately could not be cited due to constraints of space.

\section{Manufacturers' addresses}

$200{ }^{1}$ Abcam, Cambridge, Cambridgeshire, CB2 0AX, United Kingdom.

${ }^{2}$ Cell Signaling Technology, Danvers, MA, 01923, United States of America.

${ }^{3}$ Vector Laboratories, Peterborough, Cambridgeshire, PE2 6XS, United Kingdom.

\section{References}

205

206

207

208

209

210

Borges, V. F., Lyons, T. R., Germain, D. and Schedin, P. (2020) Postpartum Involution and Cancer: An Opportunity for Targeted Breast Cancer Prevention and Treatments? Cancer Res. 80, 1790-1798.

Boyce, S. D. and Goodwin, S. L. (2017) Mammary gland neoplasia in a Canadian mare: Challenges of diagnosis and treatment in a rural setting. Can. Vet. J. 58, 628-630.

Bussche, L., Harms, C., Buckles, E. L., Whelchel, D., Brosnahan, M. and Van De Walle, G. R. (2017) Carcinoma of the mammary gland in a mare. Equine Vet. Educ. 29, 370375.

Canisso, I. F., Podico, G. and Ellerbrock, R. E. (2019) Diagnosis and treatment of mastitis in mares. Equine Vet. Educ. Epub ahead of print. https://doi.org/10.1111/eve.13228

Dagher, E., Abadie, J., Loussouarn, D., Campone, M. and Nguyen, F. (2019) Feline Invasive Mammary Carcinomas: Prognostic Value of Histological Grading. Vet. Pathol. 56, $660-670$. 
219 Dontu, G. and Ince, T. A. (2015) Of mice and women: a comparative tissue biology

220

221

222

223

224

225

226

227

228

229

230

231

232

233

234

235

236

237

238

239

240

241

242

243 perspective of breast stem cells and differentiation. J. Mammary Gland Biol. Neoplasia. 20, 51-62.

Elston, C. W. and Ellis, I. O. (1991) Pathological prognostic factors in breast cancer. I. The value of histological grade in breast cancer: experience from a large study with long-term follow-up. Histopathology. 19, 403-10.

Freeman, K. P. (2002) Cytological evaluation of the equine mammary gland. Equine Vet. Educ. 14, 97-98.

Goldschmidt, M. H., Pena, L. and Zappulli, V. (2017) Tumors of the mammary gland. In: Tumors in domestic animals. $5^{\text {th }}$ edn., Ed: D. J. Meuten, John Wiley \& Sons Inc., Ames, Iowa, USA. pp 723-765.

Gusterson, B. and Eaves, C. J. (2018) Basal-like Breast Cancers: From Pathology to Biology and Back Again. Stem Cell Rep. 10, 1676-1686.

Gusterson, B. A., Ross, D. T., Heath, V. J. and Stein, T. (2005) Basal cytokeratins and their relationship to the cellular origin and functional classification of breast cancer. Breast Cancer Res. 7, 143-8.

Hirayama, K., Honda, Y., Sako, T., Okamoto, M., Tsunoda, N., Tagami, M. and Taniyama, H. (2003) Invasive ductal carcinoma of the mammary gland in a mare. Vet. Pathol. 40, 86-91.

Hitchcock, J. R., Hughes, K., Harris, O. B. and Watson, C. J. (2020) Dynamic architectural interplay between leucocytes and mammary epithelial cells. FEBS J. 287, 250266.

Hughes, K., Scase, T. J. and Foote, A. K. (2015) Estrogen Receptor and Signal Transducer and Activator of Transcription 3 Expression in Equine Mammary Tumors. Vet. Pathol. 52, 631-4. 
244 Kato, M., Higuchi, T., Hata, H., Ishikawa, Y. and Kadota, K. (1998) Lactalbumin-positive 245 mammary carcinoma in a mare. Equine Vet. J. 30, 358-60.

Lambe, M., Hsieh, C., Trichopoulos, D., Ekbom, A., Pavia, M. and Adami, H. O. (1994) Transient increase in the risk of breast cancer after giving birth. N. Engl. J. Med. $331,5-9$.

249

250

251

252

253

254

255

256

257

258

259

260

261

262

263

264

265

266

267

Mccue, P. M. and Wilson, W. D. (1989) Equine mastitis--a review of 28 cases. Equine Vet. J. 21, 351-3.

Mills, S. W., Musil, K. M., Davies, J. L., Hendrick, S., Duncan, C., Jackson, M. L., Kidney, B., Philibert, H., Wobeser, B. K. and Simko, E. (2015) Prognostic value of histologic grading for feline mammary carcinoma: a retrospective survival analysis. Vet. Pathol. 52, 238-49.

Munson, L. (1987) Carcinoma of the mammary gland in a mare. J. Am. Vet. Med. Assoc. $191,71-2$.

Overley, B., Shofer, F. S., Goldschmidt, M. H., Sherer, D. and Sorenmo, K. U. (2005) Association between ovarihysterectomy and feline mammary carcinoma. J. Vet. Intern. Med. 19, 560-3.

Prendergast, M., Bassett, H. and Larkin, H. A. (1999) Mammary carcinoma in three mares. Vet. Rec. 144, 731-2.

Ramos-Vara, J. A. (2017) Principles and Methods of Immunohistochemistry. Methods Mol. Biol. 1641, 115-128.

Reppas, G. P., Mcclintock, S. A., Canfield, P. J. and Watson, G. F. (1996) Papillary ductal adenocarcinoma in the mammary glands of two horses. Vet. Rec. 138, 518-9.

Sabiza, S., Rezaie, A., Naddaf, H., Dezfouli, M. S. and Niya, M. K. (2020) Surgical removal of a mammary gland comedocarcinoma in an Arabian mare; postoperative 
management, histopathological and immunohistochemical features. Equine Vet.

269

270

271

272

273

274

275

276

277

278

279

280

281

282

283

284

285

286

287

288

289

290

291

292 Educ. In press.

Shank, A. M. (2009) Mare mammary neoplasia: Difficulties in diagnosis and treatment. Equine Vet. Educ. 21, 475-477.

Stewart, T. A., Hughes, K., Hume, D. A. and Davis, F. M. (2019) Developmental StageSpecific Distribution of Macrophages in Mouse Mammary Gland. Front. Cell. Dev. Biol. 7, 250.

Subramani, R. and Lakshmanaswamy, R. (2017) Pregnancy and Breast Cancer. Prog. Mol. Biol. Transl. Sci. 151, 81-111.

Sysel, A. M., Moll, H. D., Livesey, M. A., Pringle, J. and Sloane, D. E. (1993) Mastectomy in three of six cases of equine mammary neoplasia. In: Scientific Presentation Abstracts ACVS 1993. Vet. Surg. 22, 370-405.

Taylor, G. N., Shabestari, L., Williams, J., Mays, C. W., Angus, W. and Mcfarland, S. (1976) Mammary neoplasia in a closed beagle colony. Cancer Res. 36, 2740-3.

Watson, C. J. and Miller, W. R. (1995) Elevated levels of members of the STAT family of transcription factors in breast carcinoma nuclear extracts. Br. J. Cancer. 71, 8404.

\section{Figure legends}

Figure 1. Luminal equine mammary epithelial cells weakly express cytokeratin 8/18, and basal mammary epithelial cells express alpha-smooth muscle actin. Dual immunohistochemical staining for cytokeratin 8/18 (brown; arrowhead) and alpha-smooth muscle actin (red; arrow) in non-neoplastic equine mammary tissue. The mammary epithelial bilayer is indicated by a bracket and an asterisk. Haematoxylin counterstain. Scale bar indicates $50 \mu \mathrm{m}$. 
293

294 Figure 2. Basal equine mammary epithelial cells express cytokeratin 14.

295 Immunohistochemical staining for cytokeratin 14 (brown) (Hughes et al., 2015) to delineate 296 the basal epithelial cells (arrow). Non-neoplastic equine mammary tissue. The mammary 297 epithelial bilayer is indicated by a bracket and an asterisk. Haematoxylin counterstain. Scale 298 bar indicates $50 \mu \mathrm{m}$.

299

300

301

302 


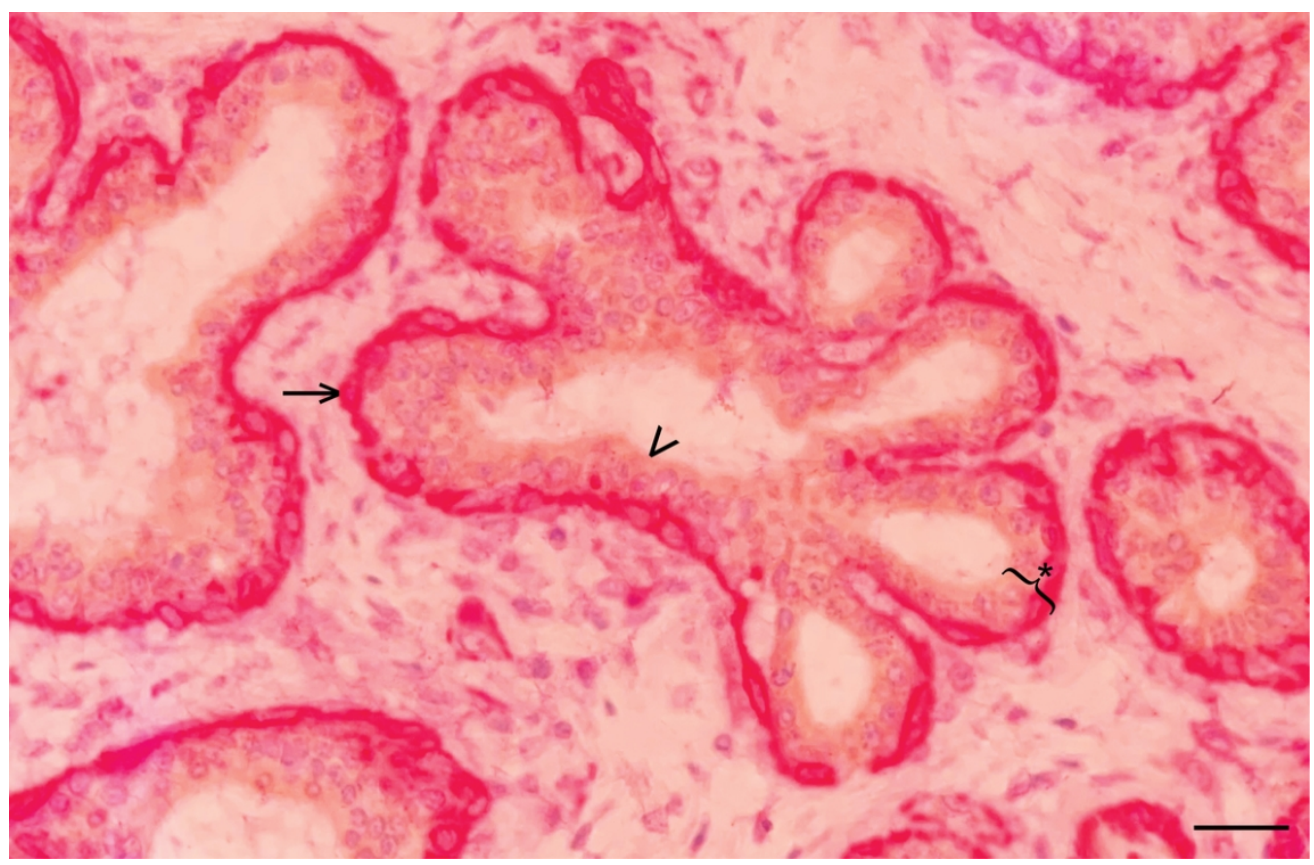

Figure 1. Luminal equine mammary epithelial cells weakly express cytokeratin $8 / 18$, and basal mammary epithelial cells express alpha-smooth muscle actin. Dual immunohistochemical staining for cytokeratin $8 / 18$ (brown; arrowhead) and alpha-smooth muscle actin (red; arrow) in non-neoplastic equine mammary tissue. The mammary epithelial bilayer is indicated by a bracket and an asterisk. Haematoxylin counterstain. Scale bar indicates $50 \mu \mathrm{m}$.

$99 \times 65 \mathrm{~mm}(300 \times 300 \mathrm{DPI})$ 


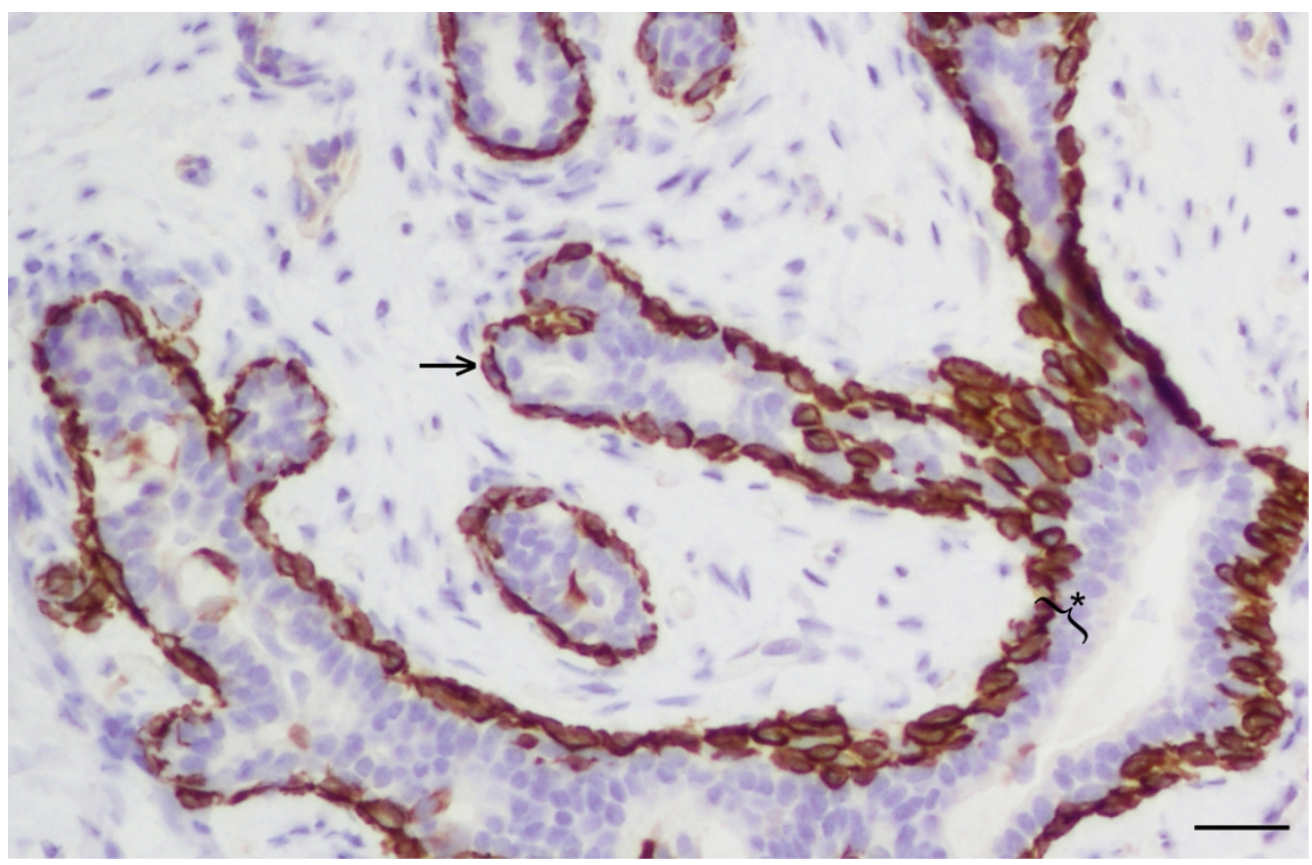

Figure 2. Basal equine mammary epithelial cells express cytokeratin 14. Immunohistochemical staining for cytokeratin 14 (brown) (Hughes et al., 2015) to delineate the basal epithelial cells (arrow). Non-neoplastic equine mammary tissue. The mammary epithelial bilayer is indicated by a bracket and an asterisk. Haematoxylin counterstain. Scale bar indicates $50 \mu \mathrm{m}$. 